\title{
Validación del cuestionario de calidad de vida (WHOQOL-BREF) en adultos mayores chilenos
}

\author{
IRIS ESPINOZA ${ }^{1, \mathrm{a},}$, PAULINA OSORIO ${ }^{2, \mathrm{~b}}$, MARÍA JOSÉ TORREJÓN ${ }^{2, \mathrm{c}}$, \\ RAMONA LUCAS-CARRASCO ${ }^{3}$, DANIEL BUNOUT ${ }^{4}$
}

\section{Validation of the WHOQOL-BREF quality of life questionnaire among Chilean older people}

\begin{abstract}
Background: The WHOQOL-BREF is a generic questionnaire to measure quality of life created by the Study Group on Quality of Life of the World Health Organization. Aim: To adapt and evaluate the psychometric properties of the Spanish version of the WHOQOL-BREF instrument in a group of Chilean older subjects living in Metropolitan Santiago. Material and Methods: A linguistic-cultural adaptation of the Spanish version of the WHOQOL-BREF was performed and tested in a pilot study. Subsequently, the modified scale was applied to a group of 1186 older women aged $72 \pm 8$ years and 334 men aged $72 \pm 7$ years. The psychometric properties such as internal consistency, item-total correlation of responses, and construct validity were evaluated. A confirmatory factor analysis was done to check if the dimensions described in the original version, were evaluated. Analyses were performed with STATA statistical software 10.0 and LISREL 8.50. Results: Confirmatory factor analysis confirmed that the dimensions physical health, psychological, social relationships and environment, described by the authors in their original description, were maintained. The instrument had a good internal consistency with a Cronbach alpha of 0.88 for the total scale and ranging from 0.70 to 0.79 in each of the dimensions. Conclusions: The evaluated version of the WHOQOL-BREF has an acceptable reliability and validity, and suggests that it is suitable for the assessment of Quality of Life in elderly people in Chile.
\end{abstract}

(Rev Med Chile 2011; 139: 579-586).

Key words: Aged; Quality of life; Questionnaires.

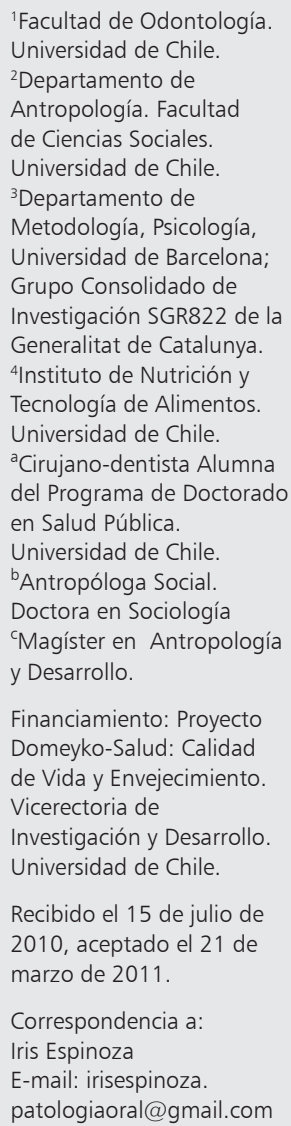

E n Chile, según el CENSO del año 2002, los adultos mayores constituían el 11,24\% de la población ${ }^{1}$, sin embargo, se estima que el año 2015 esta cifra alcanzará a 14,7\%².

Para enfrentar el desafío de satisfacer las necesidades de servicios, salud, vivienda, transporte, entre otros, de los adultos mayores es clave mejorar su calidad de vida ${ }^{3,4}$. Calidad de vida ha sido definida por la Organización Mundial de la Salud (OMS) como la percepción del individuo sobre su posición en la vida, en el contexto de la cultura y el sistema de valores en que vive, en relación con sus objetivos, expectativa, estándares y preocupaciones ${ }^{5}$.

En la década de 1990-99 la OMS reunió un grupo de investigadores de diferentes países para obtener una definición consensuada de calidad de vida y desarrollar un instrumento para medirla que fuera aplicable transculturalmente. El primero de estos fue el WHOQOL- $100^{6}$. Posteriormente, se seleccionó la mejor pregunta de cada una de las facetas, obteniéndose el WHOQOL-BREF, que produce un perfil de calidad de vida de cuatro áreas: física, psicológica, relaciones sociales y ambiente ${ }^{7}$. 
Versiones de este instrumento en inglés, chino, checo, persa, indonesio, polaco, ruso y tailandés, pueden revisarse en la página web de la Organización Mundial de la Salud (http://www.who.int/ substance_abuse/research_tools/whoqolbref/en/).

Validaciones del WHOQOL-BREF se encuentran en la literatura en adultos mayores ${ }^{8-12}$, población general ${ }^{13} \mathrm{y}$ con problemas de salud y otras condiciones $^{14-17}$. En Chile, un estudio ha usado la versión oficial española de este instrumento, sin embargo, en él no se describe si fueron evaluadas las características psicométricas del instrumento y confianza para la aplicabilidad en nuestro medio ${ }^{18}$.

El objetivo del presente estudio es evaluar las propiedades psicométricas del WHOQOLBREF en adultos mayores chilenos, información que contribuirá a analizar los resultados de las investigaciones con este instrumento en estudios nacionales. Los procedimientos de validación que se incluirán son adaptación lingüística-cultural, validez de constructo a través de análisis factorial confirmatorio y evaluación de consistencia interna.

\section{Material y Método}

\section{Sujetos y procedimientos}

La muestra estuvo formada inicialmente por 1.520 adultos mayores voluntarios que participaron en el Proyecto de Investigación: Calidad de Vida y Envejecimiento, Domeyko-Universidad de Chile.

Los participantes fueron invitados desde los programas del adulto mayor de las diferentes comunas de la provincia de Santiago. Para la selección de las comunas, estas fueron clasificadas en comunas de nivel socioeconómico alto, medio y bajo, según sus características del porcentaje de hogares pobres en la Encuesta CASEN 2006. En cada uno de los grupos de comunas se seleccionaron inicialmente al azar dos comunas para luego contactar los encargados de los Programas del Adulto Mayor y organizar la invitación de los participantes. A todos los participantes se les explicaron los fines de la investigación y a quienes accedieron a participar se les solicitó firmaran un consentimiento. La investigación fue aprobada por el Comité de Ética del Instituto de Nutrición y Tecnología de los Alimentos (INTA). El entrenamiento de los encuestadores para la aplicación del instrumento fue realizado por académicos de la Facultad de Ciencias Sociales-Universidad de Chile, quienes coordinaron el trabajo de campo.

Los procedimientos de validación de este instrumento incluyeron adaptación lingüísticacultural, validez de constructo a través de análisis factorial confirmatorio y evaluación de consistencia interna.

\section{Instrumento}

El centro WHOQOL de Barcelona, España, autorizó y facilitó el instrumento. El WHOQOLBREF tiene 26 preguntas, dos preguntas generales sobre calidad de vida y satisfacción con el estado de salud, y 24 preguntas agrupadas en cuatro áreas: Salud Física, Salud Psicológica, Relaciones Sociales y Ambiente. Puntuaciones mayores indican mejor calidad de vida. Las escalas de respuesta son de tipo Likert, con 5 opciones de respuesta.

\section{Descripción de los datos y análisis estadístico}

Se realizó un estudio descriptivo de las variables sociodemográficas de los participantes. Las variables categóricas fueron descritas en porcentajes. La distribución normal de las variables numéricas y en escala continua fue comprobada utilizando el test de Shapiro-Wilk. Las variables con una distribución normal se expresaron como promedios y desviación estándar. Aquellas sin distribución normal se expresaron como mediana y percentiles.

La validación del instrumento en primer lugar verificó si en su aplicación se distinguían las dimensiones que fueron descritas originalmente, para esto se realizó un análisis factorial confirmatorio con LISREL 8.50. Como método de extracción de factores se utilizó el método de componentes principales. Debido a las características de las respuestas de este cuestionario en una escala de tipo Likert, se procedió a obtener con PRELIS la matriz policórica que es la matriz correcta que asume que detrás de cada variable categórica hay una variable en escala. El método de estimación del modelo fue el de máxima verosimilitud.

Para evaluar la fiabilidad se analizó la consistencia interna a través del cálculo del alpha de Cronbach, que es una medida de la intercorrelación entre los ítems que conforman la escala. Un alpha de Cronbach superior a 0,7 es aceptable ${ }^{19}$.

Se consideró significativo un valor $\mathrm{p}$ menor o igual a 0,05. Los programa estadísticos utilizados para los análisis fueron: STATA 10.0 y LISREL 8.50. 
Validación del cuestionario WHOQOL-BREF en adultos chilenos - I. Espinoza et al

\section{Resultados}

\section{Adaptación del cuestionario}

El equipo de investigación revisó las definiciones sobre calidad de vida y los aspectos involucrados en la generación de este instrumento. Se revisó la redacción de las preguntas y si estas eran comprensibles para el lenguaje usado en nuestro país, teniendo presente el contenido que querían medir en su versión original. Producto de esta revisión se realizaron aclaraciones entre paréntesis a cuatro de las preguntas. A continuación se aplicó una encuesta piloto para verificar la comprensibilidad del instrumento y el tiempo promedio de aplicación.

\section{Descripción del grupo en estudio}

Se encuestaron 1.186 mujeres cuya edad era $72 \pm 8$ años y 334 hombres cuya edad era $72 \pm 7$ años. Luego del análisis exploratorio de los datos se aplicaron las recomendaciones del grupo WHOQOL. En primer lugar, 9 individuos fueron descartados debido a que presentaban menos de $20 \%$ de respuestas de la escala. De los 1.511 restantes se detectó que la gran mayoría de las preguntas presentaban un escaso número de respuestas perdidas, entre $0-13$. Sin embargo, 4 preguntas presentaron los mayores porcentajes de respuestas perdidas: ¿Hasta qué punto tiene oportunidades para realizar actividades de ocio? con 70 respuestas sin contestar $(4,6 \%)$, ¿Es capaz de desplazarse de un lugar a otro? con 69 respuestas perdidas $(4,6 \%)$, ¿Cuán satisfecho está del apoyo que obtiene de sus amigos? con 23 pérdidas $(1,5 \%)$, y principalmente la pregunta ¿Cuán satisfecho está con su vida sexual?, que presentó 405 respuestas sin contestar $(26,8 \%)$. Posteriormente, se realizó la asignación de respuestas (imputación) con el puntaje promedio de cada dimensión. Siguiendo las restricciones presentadas en la metodología, 8 encuestas más debieron ser eliminadas debido a que no fue posible calcular el puntaje de la dimensión. En resumen 17 encuestas fueron excluidas, correspondientes al 1,7\% y dejando una muestra para análisis de 1.503 participantes.

La características de la muestra final se presentan en la Tabla 1, destacando que son en un mayor porcentaje mujeres, predominantemente casadas y con educación básica.

Respecto a los puntajes por dominio obtenidos al aplicar el WHOQOL-BREF (rango posible se en- cuentra entre 4-20), se puede observar en la Tabla 2 que este fue similar en los dominios salud física, psicológica y relaciones sociales, y sólo inferior en aproximadamente un punto, en el referido a medio ambiente. En ninguna de las dimensiones el puntaje presentó una distribución normal.

En todas las dimensiones se obtuvieron puntajes máximos. El mayor porcentaje fue encontrado en relaciones sociales con 43 individuos (2,9\%), seguido por la dimensión salud física obtenido por 10 participantes $(0,7 \%)$, dimensión psicológica por 4 participantes $(0,3 \%)$ y ambiental por sólo 3 participantes $(0,2 \%)$.

\section{Análisis factorial}

El modelo que se propone como hipótesis a contrastar en este análisis factorial confirmatorio corresponde a uno que contiene 4 dimensiones: salud física, aspectos psicológicos, relaciones sociales y ambiente, según se muestra en la Tabla 3.

\section{Tabla 1. Características generales de la muestra}

\begin{tabular}{|c|c|c|}
\hline Variables & & \\
\hline Edad & & \\
\hline Promedio en años (DE) & $72(8)$ & \\
\hline & $n$ & $\%$ \\
\hline Sexo & 1.174 & \\
\hline Hombre & & 21,9 \\
\hline Mujer & & 78,1 \\
\hline Estado civil & & \\
\hline Soltero/a & 162 & 10,8 \\
\hline Casado/a / conviviente & 692 & 46,3 \\
\hline Viudo/a & 141 & 9,4 \\
\hline Separado/a & 499 & 33,4 \\
\hline Tiene pareja & & \\
\hline $\mathrm{Si}$ & 795 & 52,9 \\
\hline No & 708 & 47,1 \\
\hline Nivel educacional & & \\
\hline Analfabeto/a & 26 & 1,7 \\
\hline Básica & 440 & 29,5 \\
\hline Enseñanza media & 611 & 40,9 \\
\hline Técnica & 171 & 11,4 \\
\hline Universitaria & 246 & 16,5 \\
\hline No consignado & 9 & 0,6 \\
\hline Nivel socioeconómico de & & \\
\hline Alto & 496 & 33 \\
\hline Medio & 257 & 17,1 \\
\hline Bajo & 750 & 49,9 \\
\hline
\end{tabular}


Tabla 2. Características del los puntajes obtenidos en cada una de las 4 dimensiones del WHOQOL-BREF

\begin{tabular}{|lccccccc|}
\hline Dimensión & $\begin{array}{c}\mathbf{n} \\
\text { ítems }\end{array}$ & Mediana & $\begin{array}{c}\text { Percentil } \\
\mathbf{2 5 - 7 5}\end{array}$ & $\begin{array}{c}\text { Puntaje } \\
\text { promedio }\end{array}$ & $\begin{array}{c}\text { Desviación } \\
\text { estándar }\end{array}$ & Mínimo & Máximo \\
\hline Física & 7 & 14,4 & $13,2-16$ & 14,4 & 2,4 & 5,2 & 20 \\
Psicológica & 6 & 14,8 & $13,2-16$ & 14,5 & 2,2 & 5,2 & 20 \\
Relaciones sociales & 3 & 14,8 & $12-16$ & 14,0 & 2,5 & 4,0 & 20 \\
Ambiente & 8 & 13,2 & $12-14,4$ & 13,2 & 2,1 & 6,4 & 20 \\
\hline
\end{tabular}

Tabla 3. Resultados del análisis de correlación ítem-test y consistencia interna

\begin{tabular}{|c|c|c|}
\hline Facetas & Correlación ítem-test & Alpha de Cronbach \\
\hline \multicolumn{3}{|l|}{ Salud física } \\
\hline Dolor & 0,4705 & 0,8771 \\
\hline Dependencia de medicinas** & 0,3797 & 0,8798 \\
\hline Energía para la vida diaria* & 0,6921 & 0,8694 \\
\hline Movilidad & 0,5627 & 0,8732 \\
\hline Sueño y descanso & 0,5056 & 0,8753 \\
\hline Actividades de la vida diaria* & 0,6533 & 0,8707 \\
\hline Capacidad de trabajo & 0,6164 & 0,8717 \\
\hline \multicolumn{3}{|l|}{ Psicológico } \\
\hline Sentimientos positivos* & 0,6348 & 0,8711 \\
\hline Espiritualidad, religión, creencias personales & 0,5840 & 0,8728 \\
\hline Pensamiento, aprendizaje, memoria, concentración & 0,4801 & 0,8754 \\
\hline Imagen corporal & 0,4895 & 0,8753 \\
\hline Autoestima* & 0,6380 & 0,8712 \\
\hline Sentimientos negativos & 0,4519 & 0,8771 \\
\hline \multicolumn{3}{|l|}{ Relaciones sociales } \\
\hline Relaciones personales & 0,5861 & 0,8727 \\
\hline Actividad sexual** & 0,3903 & 0,8783 \\
\hline Apoyo social & 0,4711 & 0,8759 \\
\hline \multicolumn{3}{|l|}{ Ambiente } \\
\hline Libertad y seguridad & 0,4895 & 0,8750 \\
\hline Ambiente físico & 0,4868 & 0,8753 \\
\hline Recursos económicos & 0,5165 & 0,8748 \\
\hline Oportunidad de información & 0,4886 & 0,8754 \\
\hline Ocio y descanso & 0,5387 & 0,8744 \\
\hline Hogar & 0,5298 & 0,8741 \\
\hline Atención sanitaria/social & 0,4207 & 0,8776 \\
\hline Transporte & 0,4557 & 0,8773 \\
\hline Puntaje total del WHOQOL-BREF & & 0,88 \\
\hline
\end{tabular}

*Preguntas con las más altas correlaciones item-test, valores sobre 0,6. **Preguntas con las más bajas correlaciones item-test, valores bajo 0,4. 
Estas 4 dimensiones además se correlacionarían entre sí en la evaluación general del constructo calidad de vida.

La bondad de ajuste del modelo fue testeada usando un análisis factorial confirmatorio con LISREL 8.50 y siguiendo las recomendaciones de $\mathrm{Hu} \mathrm{L}$ y Bentler $\mathrm{PM}^{20,21}$. La bondad de ajuste específica fue evaluada con la función de ajuste mínimo de $\chi^{2}=1407,47(\mathrm{p}<0,01)$ y además se utilizaron otros dos indicadores, la raíz del error cuadrático medio (RMSE) y el índice de ajuste comparativo (CFI). EL RMSE evalúa si las preguntas responden a las dimensiones, considerándose que debería rechazarse si es superior a 0,10 , en este caso el valor obtenido para este índice fue 0,056 , lo que indica que ajustan. Por otro lado, el CFI es una cantidad derivada de comparar el modelo hipotético que contenía 4 dimensiones correlacionadas con el que ocurre al aplicar el instrumento en la muestra, considerándose para este índice valores sobre 0,9 como un ajuste adecuado. En este estudio el valor obtenido fue 0,84 , levemente inferior para el valor de ajuste más adecuado, pero que se puede considerar como razonable.

En la Figura 1 se presenta un mapa conceptual construido con LISREL de las preguntas que conforman el instrumento y sus dimensiones. En ella se puede apreciar las saturaciones factoriales obtenidas para cada una de las preguntas, que en todas las dimensiones alcanzan valores mayores a 0,53 . Destaca la pregunta 10 de la dimensión salud física, ¿Tiene energía suficiente para la vida diaria? con una saturación de 0,92 , esto se puede interpretar como que esta pregunta explica una mayor proporción de la varianza.

Respecto a las correlaciones entre los factores, en el extremo derecho de este mapa conceptual con flechas bidireccionales, se muestra que tal como fue propuesto en la hipótesis las 4 dimensiones están correlacionadas.

\section{Consistencia interna}

La fiabilidad del instrumento fue muy buena, obteniéndose un alpha de Cronbach de 0,88 para el cuestionario total y para cada una de las preguntas entre $0,87-0,88$ (Tabla 3). Respecto a la evaluación de la correlación ítem-test, no se observan correlaciones inversas y destaca con alta correlación una pregunta de la dimensión física ¿Tiene energía suficiente para la vida diaria?Y dos de la dimensión psicológica ¿Cuánto disfruta de la vida? y ¿Cuán

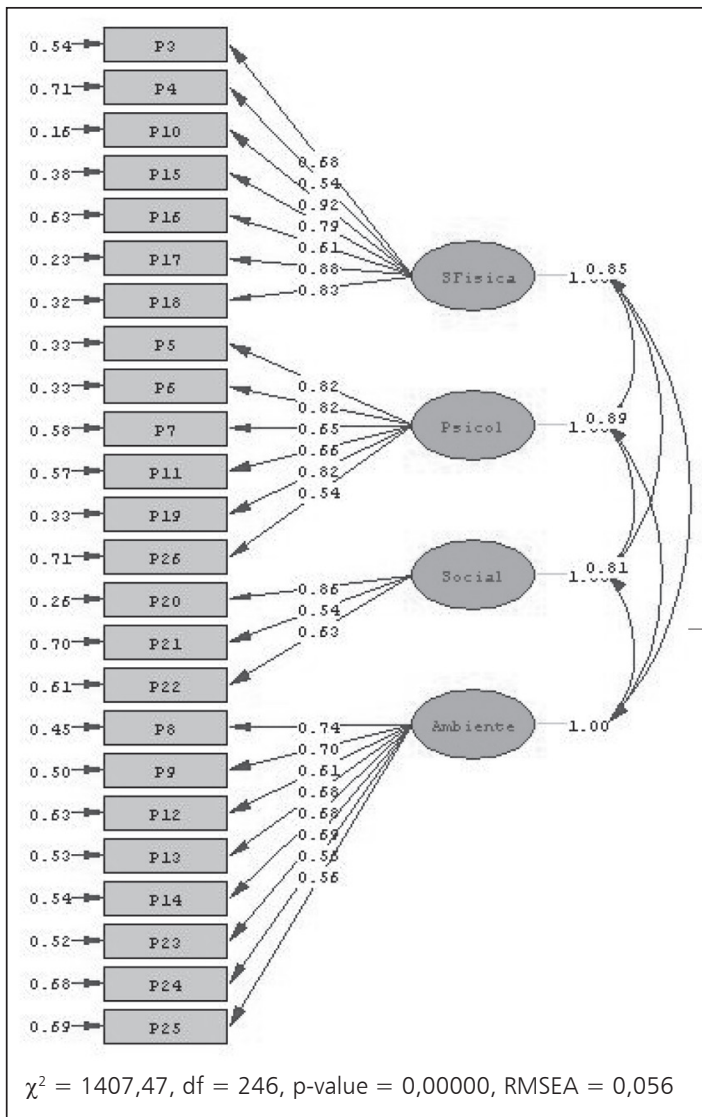

Figura 1. Modelo Conceptual de las relaciones entre las preguntas que confirman el WHOQOL-BREF y sus dimensiones.

satisfecho está de sí mismo?. Sólo dos preguntas obtienen correlaciones inferiores a 0,4 , una de estas es ¿Cuán satisfecho está con su vida sexual?.

En relación a los resultados de consistencia interna según dimensiones también se obtienen valores adecuados mayores a 0,7 (Tabla 4). La mayor correlación ítem-test se encuentra en la dimensión psicológica, que al eliminarla del cuestionario provocaba la mayor disminución de la consistencia interna.

\section{Discusión}

El presente estudio tuvo por objetivo evaluar características psicométricas del WHOQOL-BREF en una muestra de adultos mayores de Santiago. Esto debido a que se ha sugerido que para tener 
Validación del cuestionario WHOQOL-BREF en adultos chilenos - I. Espinoza et al

Tabla 4. Confiabilidad del WHOQOL-BREF en relación a su consistencia interna por dimensiones

\begin{tabular}{|lccc|}
\hline Dimensión & n de ítems & Correlación ítem-test & Alpha de Cronbach $(\mathbf{n}=\mathbf{1 . 5 0 3})$ \\
\hline Física & 7 & 0,80 & 0,74 \\
Psicológica & 6 & 0,83 & 0,70 \\
Relaciones sociales & 3 & 0,75 & 0,79 \\
Ambiental & 8 & 0,78 & 0,73 \\
Escala del test & 24 & & 0,88 \\
\hline
\end{tabular}

certeza de la validez de un instrumento en un nuevo idioma o cultura, es necesaria una repetición del proceso de validación ${ }^{23}$.

Una de las principales limitaciones de este estudio es que no fue desarrollada una muestra aleatoria por hogares sino que participaron personas de los Programas del Adulto Mayor. Sin embargo, el proceso de selección de las comunas nos permite concluir que sus resultados identificarían percepciones sobre la calidad de vida de participantes de distintos niveles socioeconómicos. Además el gran tamaño de la muestra nos aumenta la confianza de la obtención de resultados no sesgados.

Respecto a la aplicación del cuestionario, es interesante mencionar el alto porcentaje de participantes que respondió la gran mayoría de las preguntas de la escala, y que sólo fue necesario eliminar 17 individuos para los análisis. Esto nos permite indicar que este instrumento es comprensible y aplicable en nuestro medio.

Luego del estudio piloto se decidió no utilizar un formato de encuesta autoadministrada sino a través de un encuestador entrenado para evitar pérdidas de respuestas especialmente de individuos con problemas visuales o que no portaban sus anteojos. Esta decisión se justificó en la alta prevalencia de problemas de visión cercana descrita en la población chilena de adultos mayores, estimada en 91,8\% entre los individuos de 65 años y más, y además en el posible inconveniente de encontrar adultos mayores que no supieran leer, estimado en $18,6 \%$ de los individuos de este mismo grupo de edad, según la Encuesta Nacional de Salud $2003^{24}$.

Se destaca que a pesar de que se utilizó un entrevistador las omisiones fueron altas en algunas respuestas, especialmente en la referente a la satisfacción con su vida sexual. Estudios previos también han obtenido un alto porcentaje de res- puestas omitidas en esta pregunta, pero nuestros resultados son mayores al $6 \%$ observado en el estudio de la OMS en población general ${ }^{7}$. En adultos mayores la omisión de esta respuesta es aun más alta $^{12,27}$. Una posible explicación es que el tema de sexualidad todavía es tabú en la población chilena y el hecho de que la sexualidad de los mayores es objeto de variados mitos ${ }^{25}$, siendo que la realidad es que el potencial sexual puede permanecer hasta la muerte, incluso si es alterado por los cambios propios del envejecimiento ${ }^{22}$. Representaciones sociales que otorgan un carácter negativo a la sexualidad en los mayores pueden haber cohibido a los participantes para responder esta pregunta. Además, para participantes que no tienen pareja es difícil contestar esta pregunta, aunque una persona puede no tener relaciones y estar satisfecha igualmente. Es posible que esta pregunta hubiese sido respondida por una proporción mayor de adultos mayores si la encuesta hubiese sido auto administrada. Sin embargo, esta habría sido la única ventaja de no usar encuestadores. Considerando los problemas visuales y de lecto escritura que tienen muchos adultos mayores en Chile, era indispensable contar con encuestadores para obtener buenas respuestas a la encuesta.

En el análisis de la aplicabilidad de este instrumento en adultos mayores es interesante mencionar un cuestionario complementario al WHOQoL-BREF descrito por Power y $\mathrm{cols}^{27}$ que incluye temas como la muerte y el proceso de morir, el concepto psicológico de autonomía, y aspectos sobre declinación sensorial y limitaciones físicas propias de la edad ${ }^{27}$. Este instrumento WHOQoL-OLD ha mostrado buenas propiedades psicométricas en su versión española ${ }^{11}$ pero no ha sido validado en Chile.

El análisis factorial confirmatorio comprobó que en esta muestra de adultos mayores chilenos 
el instrumento WHOQOL-BREF mantiene las dimensiones Salud Física, Psicológica, Social y Ambiente, que fueron descritas en la aplicación original $^{7}$. La confirmación del modelo nos da confianza para que estudios posteriores analicen los resultados de este instrumento por dimensiones cuando el interés sea asociar su puntaje con otras variables, por ejemplo de tipo sociodemográfico, o para determinar la efectividad de intervenciones. La constancia de estas dimensiones además apoya la impresión que las mismas percepciones del WHOQOL-BREF se encuentran en distintas culturas y en personas con diferentes estados de salud/enfermedad, requerimiento fundamental para considerar un instrumento transcultural y permitir comparar los resultados entre países ${ }^{6}$.

La fiabilidad del instrumento fue satisfactoria tanto para la escala general como para cada una de las dimensiones, con resultados similares a lo descrito en el estudio original ${ }^{7}$ y en estudios en adultos mayores ${ }^{8}$. Se destaca que el alfa de Cronbach encontrado en relaciones sociales es mayor al observado en otras evaluaciones, ya que al tener sólo tres preguntas es la dimensión que muestra la menor fiabilidad.

Finalmente, los resultados nos permiten concluir que la aplicación del WHOQOL-BREF en adultos mayores chilenos ofrece medidas fiables y estructuralmente válidas en relación al modelo propuesto inicial de evaluación de calidad de vida de la OMS. Por lo anterior, recomendamos este instrumento como adecuado para su uso en nuestro medio en estudios poblacionales y estudios clínicos.

Considerando que los programas chilenos destinados al adulto mayor han solucionado muchas de las necesidades básicas de nutrición, sanitarias y de vivienda, es importante tener en cuenta que el próximo gran desafío es mejorar la calidad de vida de este grupo etario. Encuestas como la que hemos evaluado en este estudio, nos permitirán conocer cuales son las dimensiones que más afectan la calidad de vida de las personas mayores y reorientar las acciones hacia conseguir un envejecimiento exitoso de nuestra población ${ }^{28}$.

Agradecimientos: Dra. Rosa Montaño, Departamento de Bioestadística, Escuela de Salud Pública, Universidad de Chile por su asesoría en el análisis factorial confirmatorio con LISREL 8.50 .

\section{Referencias}

1. CENSO 2010. http://www.ine.cl/cd2002/sintesiscensal. pdf (Consultado el 11 de enero de 2010).

2. Instituto Nacional de Estadística (INE). Enfoque estadístico 2007. http://www.ine.cl/canales/sala_prensa/ noticias/2007/septiembre/boletin/ine_adulto_mayor.pdf

3. WHO (World Health Organization) World Atlas of Aging, World Health Organization Center for Health development, WHO Press, Kobe pp 1-138.

4. Bowling A. Growing Older Aging Well Being. Quality of Life in Old Age. Ed McGraww-Hill, England, 2005.

5. Lucas-Carrasco R. Versión española del WHOQOL. Majadahonda (Madrid): Ergón, D.L. 1998.

6. Power M, Bullinger M, Harper A, The WHOQOL Group. The World Health Organization WHOQOL-100: tests of the universality of quality of life in 15 different cultural groups worldwide. Health Psicol 1999; 18: 495505.

7. Skevington SM, Lotfy M, O'Connell KA. The World Health Organization's WHOQOL-BREF quality of life assessment: Psychometric properties and results of the international field trial. A Report from the WHOQOL Group. Qual Life Res 2004; 13: 299-310.

8. Hei-Fen Hwang, Wen-Miin Liang, Yun-Ning Chiu, Mau-Roung Lin. Suitability of the WHOQOL-BREF for community-dwelling older people in Taiwan. Age and Ageing 2003; 32: 593-600.

9. Von Steinbuchel N, Lischetzke T, Gurny M, Eid M. Assessing quality of life in older people: psychometric properties of the WHOQOL-Bref. Eur J Ageing 2006; 2: 116-22.

10. Chachamovich E, Trentini C, Fleck MP. Assessment of the psychometric performance of the WHOQOL-BREF instrument in a sample of Brazilian older adults. Int Psychogeriatr 2006, 19: 635-46.

11. Lucas-Carrasco R, Laidlaw K, Power MJ. Suitability of the WHOQOL-BREF and WHOQOL-OLD for Spanish older adults. Ageing and Mental Health. (in press).

12. Kalfoss MH, Low G, Molzahn A.E. The suitability of the WHOQOL-BREF for Canadian and Norwegian older adults. Eur J Ageing 2008; 77-80.

13. Noerholm V, Groenvold M, Watt T, Bjorner JB, Rasmussen NA, Bech P. Quality of life in the Danish general population-normative data and validity of WHOQOLBREF using Rasch and item response theory models. Qual Life Res 2004; 13: 531-40.

14. Berlim MT, Pavanello DP, Caldieraro MA, Fleck MP. Reliability and validity of the WHOQOL BREF in a sample of Brazilian outpatients with major depression. Qual Life Res 2005; 14: 561-4. 
Validación del cuestionario WHOQOL-BREF en adultos chilenos - I. Espinoza et al

15. Asnani MR, Lipps GE, Reid ME. Utility of WHOQOLBREF in measuring quality of life in sickle cell disease. Health Qual Life Outcomes 2009; 10: 75.

16. Lucas-Carrasco R, Pascual-Sedano B, Galán I, Kulisevsky J, Sastre-Garriga J, Gómez-Benito J. Using the WHOQOL-DIS to Measure Quality of Life in Persons with Physical Disabilities Caused by Neurodegenerative Disorders. Neurodegener Dis 2011; 8: 176-86.

17. Lucas-Carrasco R, Skevington SM, Gómez-Benito J, Rejas J, March J. Using the WHOQOL-BREF in Patients with Dementia: A Validation Study. Alzheimer Dis Assoc Disord. 2011 Feb 2. [Epub ahead of print].

18. Torres M, Quezada M, Rioseco R, Ducci ME. Calidad de vida en adultos mayores pobres de viviendas básicas: Estudio comparativo mediante uso de WHOQoL-Bref. Rev Med Chile 2008; 136: 325-33.

19. Bland AM, Altman DG. Cronbach's alpha. BMJ 1997; 314: 572.

20. Hu L, Bentler PM. Cutoff criterio for fit indexes in covariance structure analyses: conventional criteria versus new alternatives. Structural Equation Modelling 1999; 6: 1-5.

21. Bentler PM. Comparative fix indexes in structural models. Psychol Bull 1999; 107: 238-46.

22. Cayo G, Flores E, Perea X, Pizarro X, Aracena A. La sexualidad en el adulto mayor y su relación con el bien- estar subjetivo. Ponencia presentada en el Congreso viejo y viejas, participación ciudadana e inclusión social. 51 Congreso Internacional de Americanistas. 14 al 18 de Julio de 2003. Búsqueda 17 de enero de 2011. Documento publicado en extenso en la Biblioteca del Congreso Nacional de Chile: http://www.bcn.cl/carpeta_temas_profundidad/temas_profundidad.2008-04-30.0122799562/ documentos_pdf.2008-04-30.1359051441.

23. Guyatt GH, Feeny DH, Patrick D. Measuring HealthRelated Quality of Life. Gordon H. Ann Intern Med 1993; 118: 622-9.

24. Ministerio de Salud Chile. I Encuesta de Salud Chile, 2003. Búsqueda 23 de enero de 2010. http://epi.minsal. cl/epi/html/invest/ENS/InformeFinalENS.pdf

25. Barrientos J, Páez D. Psychosocial Variables of Sexual Satisfaction in Chile. J Sex Marital Ther 2006; 32: 351-68.

26. Arslantas D, Unsal A, Metintas S, Koc F, Arslantas A. Life quality and daily life activities of elderly people in rural areas, Eskişehir (Turkey). Arch Gerontol Geriatr 2009; 48: 127-31.

27. Power M, Quinn K, Schmidt S, and WHOQOL-OLD group. Development of the WHOQOL-Old module. Qual Life Res 2005; 14: 2197-214.

28. Rowe JW, Kahn RL. Successful aging. Gerontologist 1997; 37: 433-40. 